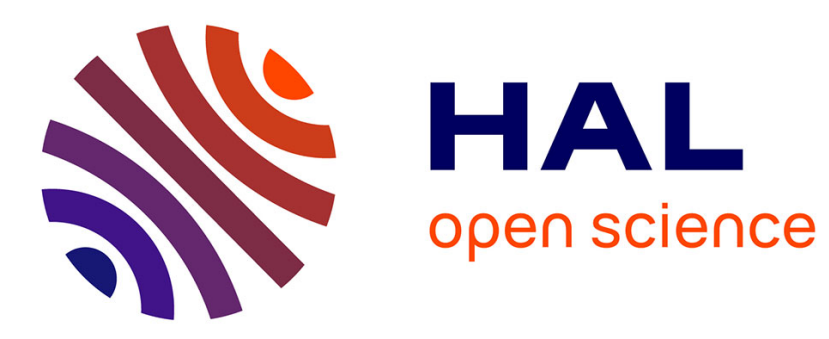

\title{
The method of a floating frame of reference for non-smooth contact dynamics
}

Alexander Lozovskiy, Frédéric Dubois

\section{To cite this version:}

Alexander Lozovskiy, Frédéric Dubois. The method of a floating frame of reference for nonsmooth contact dynamics. European Journal of Mechanics - A/Solids, 2016, 58, pp.89 - 101. 10.1016/j.euromechsol.2016.01.007 . hal-01508684

\section{HAL Id: hal-01508684 \\ https://hal.science/hal-01508684}

Submitted on 14 Apr 2017

HAL is a multi-disciplinary open access archive for the deposit and dissemination of scientific research documents, whether they are published or not. The documents may come from teaching and research institutions in France or abroad, or from public or private research centers.
L'archive ouverte pluridisciplinaire HAL, est destinée au dépôt et à la diffusion de documents scientifiques de niveau recherche, publiés ou non, émanant des établissements d'enseignement et de recherche français ou étrangers, des laboratoires publics ou privés. 


\title{
The method of a floating frame of reference for non-smooth contact dynamics
}

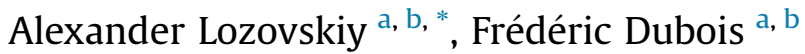 \\ ${ }^{a}$ Laboratoire de Mécanique et Génie Civil (LMGC), Université de Montpellier, CNRS, Montpellier, France \\ ${ }^{\mathrm{b}}$ Laboratoire de Micromécanique et d'Intégrité des Structures (MIST), Université de Montpellier, CNRS, IRSN, France
}

\begin{abstract}
A method of a floating frame of reference that performs splitting of a deformable solid into rigid and deforming parts is presented within the context of non-smooth contact dynamics. The decomposition is made in such a way that the deforming part of the velocity field does not contribute either to the motion of the center of mass or the rotational motion. The corresponding numerical method that computes both rigid and deforming motions is presented and extended to multi-body dynamics simulation allowing non-smooth contact interactions, such as impacts and friction. Numerical experiments, where the method is compared with a more traditionally used Total Lagrangian method, justify its preference as a more efficient tool for the simulation of assemblies of stiff and massive objects.
\end{abstract}

Keywords:

Floating frame of reference; Velocity decomposition; Non-smooth contact dynamics; Corotational formulation

\section{Introduction}

This paper presents and studies the application of the method of a floating frame of reference to a simulation of solids, participating in non-smooth dynamics.

The method of a floating frame of reference (for simplicity, it shall be referred to as FFR) is a special type of a general family of so called corotational methods. The key idea behind a corotational method is a kinematical splitting into two of the reference configuration of an element of a structure primarily discretized with the Finite Element method (FEM). These are the base configuration and the corotated, or dynamic one. The base configuration is kept fixed for the entire structural analysis, while the corotated configuration is a result of the rigid body motion, i.e. superposition of translation and rotation, of the base configuration. In general, the dynamic configuration is element dependent and is defined for each element separately. A far from complete list of the works on the topic includes (Crisfield, 1990; Bergan and Horrigmoe, 1976; Rankin and Brognan, 1986; Rankin and Nour-Omid, 1988; Belytschko and Hsieh, 1973; Simo, 1985; Devloo et al., 2000; Areias et al., 2011; Alsafadie et al., 2010; Felippa and Haugen, 2005).

\footnotetext{
* Corresponding author. Department of Mathematics, Texas A\&M University, College Station, USA.

E-mail addresses: lozovskiy@math.tamu.edu (A. Lozovskiy), frederic.dubois@ univ-montp2.fr (F. Dubois).
}

The FFR method differs significantly from general corotational methods by the fact that it requires only one dynamic configuration for each element. Even more, this single moving frame of reference is introduced without any connection to the elements or any other type of structural discretization of the described solid, and therefore can be defined before even considering discretization in general. The FFR method is intuitively more attractive and has been known for over a century. It has been mostly used for computations in flexible multi-body dynamics, where separate solids are connected via bilateral constraints, typically smooth, (Cardona and Geradin, 2001), (Veubeke, 1976). A brief overview of the method in this area was given in (Shabana et al., 2007).

Variants of the FFR method are distinguished by the type of attachment between the moving frame and the deformable body itself. This is governed by the reference conditions (Schwertassek et al., 1999). Depending on the selected reference conditions, the translational and rotational coordinates associated with the body frame vary differently during the motion. The motion of the selected frame is referred to as reference motion. For example, reference conditions result in the tangent frame, the chord frame or the free frame, among others (Escalona et al., 2003). Reference conditions are related to the boundary conditions that the selected deformation shape functions must fulfil.

Our area of interest is a simulation of assemblies of bodies undergoing non-smooth contact interactions such as shocks and friction, with typically natural external forces such as gravity. A well known method to simulate dynamics of large assemblies of objects 
with such interactions is the Discrete Element method (DEM) (Cundall and Hart, 1992). In DEM, typically only a minimal resolution of the internal deformations are performed as to allow more computational resources for the overall system dynamics. The areas of application of DEM include rocking avalanches (Banton et al., 2009), (Manzella and Labiouse, 2009), masonry structures, granular systems (Ghaboussi and Barbosa, 1990). Even the most sophisticated continuous flow models usually fail to replace DEM in representing accurate physical phenomena. As opposed to DEM, in which the number of bodies ranges from thousands to hundreds of thousands or even more (Mishra and Rajamani, 1992), we are assuming a significantly smaller number of the participating solids, though with the growing computer performance this number may naturally grow as well. The bodies are assumed to fall under an important restriction to eliminate a possibility of nonlinear deformational behavior, such as bending. Hereafter bodies are seen as massive blocks, which means no extremely thin bodies are considered. This restriction also imposes large stiffness on the participating bodies, for which the Young modulus is typically positioned around values of order $10^{9}-10^{10}$ or larger. Unlike DEM, we consider more internal degrees of freedom for the solids, which explains our limitation in the number of the solids in the multibody dynamics but allows a more accurate simulation of these solids.

This article focuses on the implementation of the FFR method in the dynamics of several bodies and its integration into the nonsmooth contact framework pioneered by J. J. Moreau (1988), (2004) and M. Jean (1999). This is a vastly growing field, and the number of methods has been developed for that framework. Unfortunately, the absolute rigidity model for interacting bodies in a studied collection as a simplification of the large stiffness model may create indeterminacy (plurality of solutions) (Alart, 2014), partly because of the specific nature of the employed interaction laws. One of the ways to treat this problem is by incorporating a finite yet large stiffness for the bodies, and therefore applying the FEM analysis. This bears the most general solver relying on the Total or Updated Lagrangian approach, also known in the engineering community as the method of a large transformation and employing the absolute nodal coordinate formulation (ANCF). The method without any regard to contact is thoroughly described in (Belytschko et al., 2000) and its implementation into contact problems may be found in (Acary and Brogliato, 2008), (Koziara and Bicanic, 2008). With an assumption of a small rotation, the nonlinearity of the method is neglected, and it is renamed into the method of small deformation.

Within the FFR framework, we shall be using the so-called deformable body mean axis frame (Agrawal, 1984). When using this frame, the degree of coupling between the reference coordinates and the elastic coordinates is minimum, though not zero. Neither the corotational method, nor the large transformation approach seem as attractive for the non-smooth dynamics of stiff massive solids as the FFR method with this frame. Assume the solid's position field satisfies $\mathbf{x}=\mathbf{x}(\mathbf{X})$, where $\mathbf{X}$ and $\mathbf{x}$ reside in the base and current configuration respectively. Then the transformation gradient $\mathbb{F}$ may be polarly decomposed as

$\mathbb{F}=\frac{\partial \mathbf{x}}{\partial \mathbf{X}}=\mathbb{U} \mathbb{O}$

where $\mathbb{U}$ is responsible for the deformation, and $\mathbb{O}$ is an orientation as of a rigid body. The assumption for our case implies $\mathbb{D} \approx$ const for the entire structure, therefore the general corotational method, operating with arbitrarily changing $\mathbb{O}$ from element to element, is overused. On the other hand, the large transformation approach is not efficient computationally, due to the presence of the high nonlinearity in the equations even in the absence of contact interactions. The floating frame is intended for tracking the rotational part of the solid, and the nonlinear deformations in this case are completely eliminated from the point of view of that frame, which allows for constant stiffness matrix in the computations throughout the whole simulation. The remaining nonlinearity is only due to rotations, which relaxes an iterative solution process.

Another attractive property of the FFR method is that the floating frame, described above, may provide such characteristics of the moving solid as its center of mass, orientation and angular velocity (without operating with rigid body modes, coming from the FEM discretization), which are the parameters of a purely rigid body and are desirable for intuitive description of deformable, but very stiff solids. For this reason, from now on we shall be referring to the motion of such frame as "rigid motion" of the deformable solid as opposed to more general term "reference motion".

The paper has 4 sections following. As a theoretical base, Section 2 provides a formal kinematical theory for the most general body structure. It is shown that the decomposition into the rigid and the deforming motions for a single solid is always possible in the kinematical sense regardless of the dynamical reasoning for the motion. Although theoretically this splitting is possible for any kind of deforming solids, computationally it is only meaningful in the application area mentioned above, as too much deformation would create nonlinearity and the FFR method would not retain its advantageous status over the method of a large transformation. Note this section is not operating with any sorts of spatial discretizations of a solid and is based purely on the fundamental laws of mechanics. This section may present interest especially for theoretical mechanicians. Section 3 introduces FEM method in the local frame to resolve the deformational behavior and derives a stable secondorder accurate Newmark time-stepping scheme and then adapts these results to the non-smooth contact dynamics of a general multi-body system. Finally, Section 4 contains numerical experiments that test the performance and accuracy of the method compared to the large transformation method. Section 5 provides the conclusion and further prospects.

\section{The formalism}

Everywhere below any bold symbol, for example a, describes a vector with at least two components or a set of vectors. Blackboard bold symbols, except for the real number set $\mathbb{R}$, denote operators, tensors (wider than vectors) and their corresponding matrices. Symbol $\times$ denotes vector product and $\otimes$ denotes tensor product of two vectors, i.e. $\mathbf{a} \otimes \mathbf{b}=\mathbf{a} \mathbf{b}^{T}$. Operation $\mathbb{A}: \mathbb{B}$ returns the sum of all products of the respective elements of the both matrices, i.e. $\sum_{i, j} A_{i j} B_{i j}$

\subsection{Kinematics}

Let the Euclidean system of coordinates Oxyz be an inertial frame of reference (Landau and Lifshitz, 1976) called a global frame. For a solid with density $\rho$ and mass $m$, let $\mathbf{x}(\mathbf{X}, t)$ denote a position vector in the global frame of a material point with Lagrange coordinates $\mathbf{X}$ at time $t$. $\mathbf{v}(\mathbf{X}, t)$ denotes the velocity vector of that point. The set of all $\mathbf{X}$ is denoted $V$ and they refer to the initial undeformed (rigid) configuration of the solid.

The main idea behind a floating frame is that any motion of a deformable body $V$ may be described as the superposition of the motions of the imaginary rigid and deforming parts (Shabana and Schwertassek, 1998). For a currently used floating orthonormal frame $C X Y Z$ and an imaginary rigid body frozen in it, with its center of mass placed at the origin $C$, the velocity $\mathbf{v}_{R}$ of that rigid body is 
completely defined by the motion of the floating frame. With the deforming velocity denoted as $\mathbf{v}_{D}$, the actual velocity satisfies

$$
\mathbf{v}=\mathbf{v}_{R}+\mathbf{v}_{D}
$$

So once a rigid part $\mathbf{x}_{R}(\mathbf{X}, t)$ and its corresponding floating frame, also called local, or rigid frame, are specified in any desired way, the equality (1) uniquely defines $\mathbf{v}_{D}$ for a provided motion of the actual solid with velocity $\mathbf{v}$.

The orientation of $C X Y Z$ is given by an orthogonal matrix $\mathbb{O}(t)$. $\mathbf{r}(\mathbf{X}, t)$ denotes a vector, expressed in the global frame, from the center of mass of the rigid part to the point of the same with material coordinates $\mathbf{X}$. By definition of $\mathbb{O}(t)$,

$\mathbf{r}(\mathbf{X}, t)=\mathbb{O}(t) \mathbf{X}$.

Let $\rho_{R}$ denote the density of the rigid part, i.e. the density of the actual solid once it fully coincides with its rigid part (density in its initial configuration). For every rigid body moving continuously in space, there exists a two-way correspondence between $\mathbf{v}_{R}$ and the velocity of the center of mass $\mathbf{v}_{R, C}$ and its angular velocity $\omega$ (Goldstein et al., 2014). This correspondence is based on linear operators $\mathbb{L}$ and $\mathbb{G}$, acting from a vector field to a single vector and in the opposite direction respectively:

$\mathbb{L}: \mathbf{v}_{R} \rightarrow\left(\begin{array}{c}\mathbf{v}_{R, C} \\ \omega\end{array}\right), \mathbb{G}:\left(\begin{array}{c}\mathbf{v}_{R, C} \\ \omega\end{array}\right) \rightarrow \mathbf{v}_{R}$

With $\rrbracket=\int \rho_{R}\left(\left(\mathbf{r}^{T} \mathbf{r}\right) \llbracket-\mathbf{r} \otimes \mathbf{r}\right) d v$ denoting the inertia tensor of the rigid part in the global axes at the center of mass (so called Koenig's frame of reference) and being diagonal in the local frame, the operator $\mathbb{L}$ has a form

$\mathbb{L}=\left(\begin{array}{c}m^{-1} \int_{V} \rho_{R} \cdot(\cdot) d v \\ \mathbb{J}^{-1} \int_{V} \rho_{R} \mathbf{r} \times(\cdot) d v\end{array}\right)$.

Note the Koenig's frame implies time-dependence of $\mathbb{J} \mathbb{G}$ is simply another way of writing Euler's formula

$\mathbf{v}_{R}=\mathbf{v}_{R, C}+\omega \times \mathbf{r}$.

It is obvious that $\mathbb{G} \mathbb{Q} \mathbf{v}_{R}=\mathbf{v}_{R}$.

We are interested in a condition

$\llbracket \mathbf{v}_{D}=0$,

which physically implies that the total momentum and the angular momentum of the deformation field of interest are zero.

Theorem 1. For every continuously moving body, there always exists such a decomposition into the rigid and the deforming parts that $\llbracket \mathbf{v}_{D}=0$ throughout the entire motion of the body. The decomposition is unique, up to the initial configuration of the rigid part.

\section{Proof.}

Indeed, specify the rigid part in any desired way at the initial instant $t=0$ (only preserving the isomorphism between $\mathbf{x}$ and $\mathbf{x}_{R}$ ). Its velocity is defined via $\mathbf{v}_{R}=\mathbf{v}_{R, C}+\omega \times \mathbf{r}$. Since the field $\mathbf{r}$ is fixed (it defines the rigid part that we constructed), we only need to adjust two vectors $\mathbf{v}_{R, C}$ and $\omega$ in the way we see appropriate. Setting

$\mathbf{v}_{R, C}=m^{-1} \int_{V} \rho_{R} \mathbf{v} d v$
$\omega=\mathbb{J}^{-1} \int_{V} \mathbf{r} \times \rho_{R} \mathbf{v} d v$

immediately satisfies (4). Once we set the rigid velocity in the way described above at $t=0$, this decomposition will preserve condition (4) for the whole motion if we simply force conditions (5) and (6) for the rigid part to fulfil.

Remark 1. The theorem may also be proven with use of the fact that the set $\mathbf{V}$ of all fields $\mathbf{v}$ for a fixed instant of time is a Hilbert space with the inner product $\langle\cdot, \cdot\rangle=\int_{V} \rho_{R}(\cdot)^{T}(\cdot) d v$. The set $\mathbf{V}_{R}=\left\{\mathbf{v}_{R} \mid \mathbf{V}_{R}=\mathbf{v}_{R, C}+\omega \times \mathbf{r}\right\}$, parametrized by $\mathbf{v}_{R, C}$ and $\omega$, is a closed linear subspace of $\mathbf{V}$ and $\mathbb{G} \mathbb{L}$ is a projection operator from $\mathbf{V}$ onto $\mathbf{V}_{R}$. By properties of a Hilbert space (Debnath and Mikusinski, 2005), there exists a unique element $\mathbf{v}_{R} \in \mathbf{V}_{R}$ such that $\mathbb{G} \mathbb{L}\left(\mathbf{v}-\mathbf{v}_{R}\right)=0$, i.e. $\llbracket \mathbf{v}_{D}=0$, since $\operatorname{Ker} \mathbb{G}=0$.

Starting now, for each solid we shall consider such a rigid part from an infinite set of those satisfying (4), that it fully coincides with the initial configuration $V$ of the solid at the initial instance. Physically, condition (4) implies that the bulk behavior preserves the motion of the center of mass of the whole system and its rotational momentum. For this reason, $\mathbf{v}_{R, C}$ is naturally replaced with $\mathbf{v}_{C}$, for shorter notation. Due to (1) and (4),

$\mathbb{G} \mathbb{L} \mathbf{v}=\mathbf{v}_{R}$ and $(\mathbb{\square} \mathbb{G} \mathbb{L}) \mathbf{v}=\mathbf{v}_{D}$.

The following holds.

\section{Proposition 1.}

$\int_{V} \rho_{R} \mathbf{v}_{R}^{T} \mathbf{v}_{D} d v=0$.

Proof. The statement of the proposition is obtained immediately by applying the argument of Remark 1 .

Remark 2. Theorem 1 lets decompose the total kinetic energy of the system into the kinetic energy of the rigid motion and of the deforming one:

$T=\frac{1}{2} \omega^{T} \rrbracket \omega+\frac{1}{2} m v_{C}^{2}+\frac{1}{2} \int_{V} \rho_{R} \mathbf{v}_{D}^{T} \mathbf{v}_{D} d v$.

Coordinate function $\boldsymbol{x}^{\prime}(\mathbf{X}, t)$ describes the actual motion of the points of the body with respect to the frame $C X Y Z$ (local, or relative motion). The set of all outputs $\boldsymbol{x}^{\prime}(\mathbf{X})$ is denoted as $V^{\prime}$. With local velocity $\mathbf{v}^{\prime}=\dot{\mathbf{x}}^{\prime}$, the Galilean addition of velocities in the global frame (Landau and Lifshitz, 1976; Goldstein et al., 2014) reads as

$\mathbf{v}=\mathbf{v}_{C}+\mathbb{O} \mathbf{v}^{\prime}+\omega \times \mathbb{O} \mathbf{x}^{\prime}$.

From here, it follows that

$\mathbf{v}_{D}=\mathbb{O}\left(\mathbf{v}^{\prime}+\mathbb{O}^{T} \omega \times \mathbf{d}^{\prime}\right)$

in the global frame with local displacement $\boldsymbol{d}^{\prime}=\boldsymbol{x}^{\prime}-\mathbf{X}$.

\subsection{The orientation of the rigid frame is not intuitive}

An interesting fact about the presented decomposition is that for systems with small stiffness it provides a rigid frame whose position intuitively does not seem obvious. Consider for instance a discrete system made of 4 material points of unit mass placed at the corners of a square and connected with each other through springs of equal stiffness $k$. For the initial conditions, let every mass point 
have a unit velocity orthogonal to the diagonal of the square that the point belongs to in such a way, that the system rotates clockwise around the center of the square. The symmetry of the problem's geometry lets us construct a moving orthonormal frame of reference that we denote $C \overline{X Y}$ such that the mass points are moving only along the axes $C \bar{X}$ and $C \bar{Y}$, see Fig. 1 .

If $\mathbf{e}_{\bar{X}}, \mathbf{e}_{\bar{Y}}$, and $\mathbf{e}_{\bar{Z}}$ denote the standard basis of $C \overline{X Y Z}$ (axis $C \bar{Z}$ is orthogonal to the plane $C \overline{X Y}$ ), then the position vector of one of the 4 points is $\mathbf{r}=r \mathbf{e}_{\bar{X}}$ with a scalar function $r=r(t)$. This frame rotates with some time-dependent angular velocity $\omega_{1}=\dot{\phi}_{1} \mathbf{e}_{\bar{Z}}$ and acceleration $\dot{\omega}_{1}=\ddot{\phi}_{1} \mathbf{e}_{\bar{z}}$, where angle $\phi_{1}$ describes the orientation of this frame with respect to the global one. The velocity of the point is obtained by direct differentiation as $\mathbf{v}=\dot{\boldsymbol{r}} \mathbf{e}_{\bar{X}}+r \omega_{1} \mathbf{e}_{\bar{Y}}$. The first term on the right-hand side is non other than the local velocity of the point with respect to the frame $C \overline{X Y}$. Then momentum equation for this mass point with respect to the same frame is then

$\ddot{r} \mathbf{e}_{\bar{X}}=4 k(1-r) \mathbf{e}_{\bar{X}}+\omega_{1}^{2} \mathbf{r}-\dot{\omega}_{1} \times \mathbf{r}-2 \omega_{1} \times \dot{r} \mathbf{e}_{\bar{X}}$.

The last two vector terms on the right-hand side (Euler and Coriolis forces) are parallel to the $C \bar{Y}$-axis. The fact that the material point never leaves axis $C \bar{X}$ implies that these forces cancel each other, so we obtain $\omega_{1} \dot{r}=-2 \omega_{1} \dot{r}$. From here, $\omega_{1}=r^{-2}$. So the final differential equation for $r$ then will be

$\ddot{r}=4 k(1-r)+r^{-3}$

which we have to solve with initial conditions $r(0)=1$ and $\dot{r}(0)=0$. Interestingly, the frame $C \overline{X Y}$ is not a frame of the ridid part that fulfils $\llbracket \mathbf{v}_{D}=0$. Therefore, there must exist, according to Theorem 1 , another frame $C X Y$ rotating with angular velocity $\omega=\dot{\phi} \mathbf{e}_{\bar{Z}}$ such that condition $\llbracket \mathbf{v}_{D}=0$ holds for that frame. Let $\mathbf{v}_{R}=\omega \mathbf{e}_{Y}$ denote the velocity of the rigid part at the first mass point. For the condition $\llbracket \mathbf{v}_{D}=0$ to hold, it is necessary and sufficient to require, due to symmetry, that $\mathbf{v}-\mathbf{v}_{R}$ be orthogonal to $C Y$-axis. This is equivalent to

$\left(\dot{r} \mathbf{e}_{\bar{X}}+r \omega_{1} \mathbf{e}_{\bar{Y}}-\omega \mathbf{e}_{Y}\right) \cdot \mathbf{e}_{Y}=0$.

If we denote the angle between two frames $C \overline{X Y}$ and $C X Y$ as $\alpha(t)=\phi(t)-\phi_{1}(t)$, then we finally obtain our goal differential equation

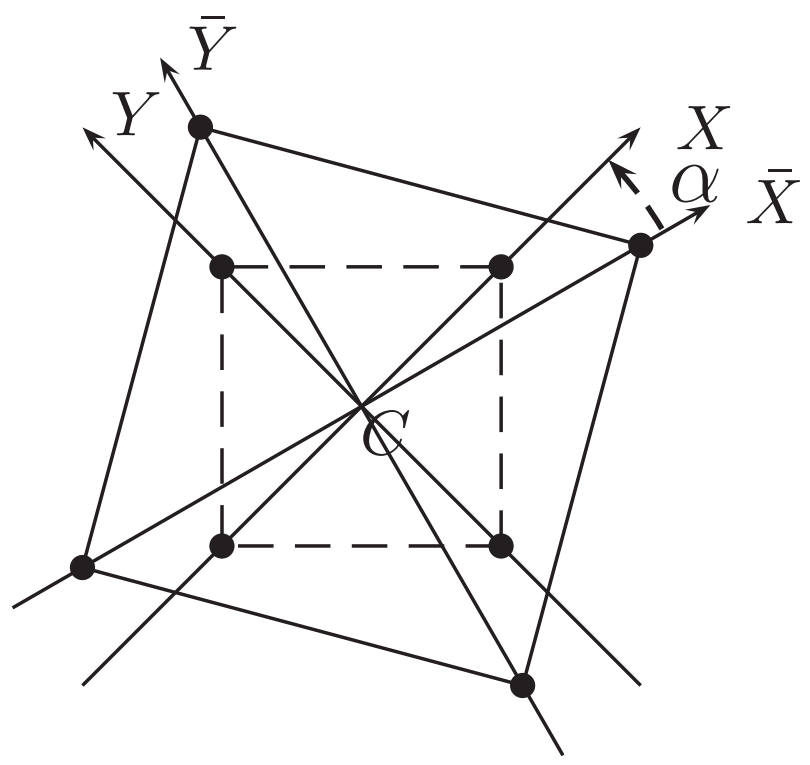

Fig. 1. The system itself (outer square) and its rigid component (inner square). $\dot{\alpha}=-\dot{r} \sin \alpha+r^{-1} \cos \alpha-r^{-2}$.

Numerical solution of this equation with initial condition $\alpha(0)=0$ shows that $\alpha$ always stays positive (meaning that the frame $C X Y$ lags behind $C \overline{X Y}$ ), although does not grow to infinity. It stabilizes after some time and oscillates around some constant value. The more stiff the system is, with the rest of input being the same, the smaller $\alpha$ becomes, so the closer the two frames approach each other. Refer to graphs of Fig. 2. The limit at infinity of the mean value of $\alpha$ may be estimated from solving the equilibrium equation $r^{-1} \cos \alpha-r^{-2}=0$. This value is estimated to be $\alpha_{\infty}=\arccos \left(r_{\infty}^{-1}\right)$, where $r_{\infty}$ is a solution of equation $4 k(1-r)+$ $r^{-3}=0$. Table 1 shows the limit of the mean value of $\alpha$ depending on $k$.

The presented example demonstrates the presence of the drift between intuitively desirable frame and the actual frame $C X Y$ granting conservation of linear and angular momentums of the deforming motion. However, for the applications that this works aims at, the stiffness is large enough for this kind of drift to remain unnoticable, as is confirmed by the example above. Therefore, in the upcoming study, we will be proposing to treat the deforming velocity $\mathbf{v}_{D}$ as $\mathbb{O} \mathbf{v}^{\prime}$, meaning that the geometrical nonlinearities (caused by rotation) with respect to the local frame are negligible. This will allow to work with constant in time stiffness matrix in the local frame. The justification of such simplification in the numerical scheme for more practical simulations will be confirmed by the numerical experiments. It will also be shown how this simplification allows to incorporate dynamics into the contact framework.

\subsection{Dynamics}

The force vectors acting on a body are described as internal, i.e. those acting from the points of the body itself, and external forces (Landau and Lifshitz, 1976). They are denoted $\mathbf{F}_{\text {int }}$ and $\mathbf{F}_{\text {ext }}$ respectively. Note that $\mathbf{F}_{e x t}=\mathbf{F}_{e x t}(t)$ is considered here general spaceindependent volumic force, but possibly time-dependent. It is different from forces $\mathbf{R}$ acting on surfaces, such as contacts.

Now we can write the main dynamics equation governing the solid pointwise:

$\rho \dot{\mathbf{v}}=\mathbf{F}_{\text {int }}+\mathbf{F}_{\text {ext }}+\mathbf{R}$

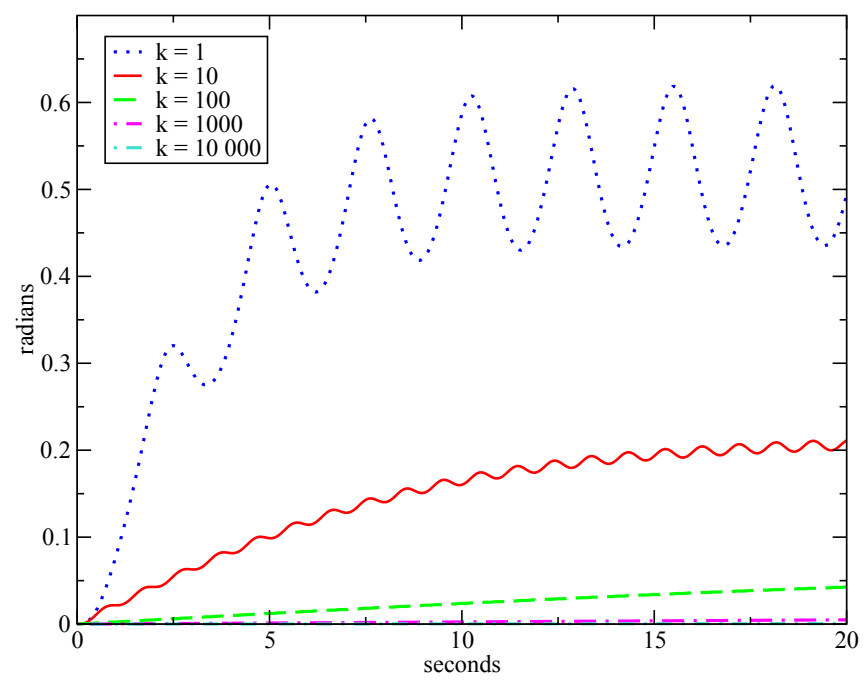

Fig. 2. Evolution of the angle $\alpha$ between the two frames for varying stiffness k. 
Table 1

The increase of stiffness $\mathrm{k}$ causes the decrease of $\alpha_{\infty}$

\begin{tabular}{llllll}
\hline$k$ & 1 & 10 & 100 & 1000 & 10000 \\
\hline$\alpha_{\infty}$ & 0.5316 & 0.2139 & 0.0704 & 0.0223 & 0.0071 \\
\hline
\end{tabular}

where $\mathbf{R}$ is taken zero everywhere outside the contact region. The trajectory of the center of mass of the whole system is described via

$\dot{\mathbf{v}}_{C}=\frac{1}{m}\left(\int_{V^{\prime}} \mathbf{F}_{e x t} d v^{\prime}+\int_{\partial V^{\prime}} \mathbf{R} d s\right)$

which is a known result. Also, according to (6), the rotational motion of the system is determined via

$\rrbracket \boldsymbol{\omega}=\int_{V^{\prime}} \mathbb{O} \mathbf{X} \times \rho \mathbf{v} d v^{\prime}$

A time discretization of this equation creates an unstable scheme, and it additionally puts computational burden on the simulation, which comes from the need of integration over the entire solid. Instead, (12) must be differentiated in time. This provides an equation, which is too heavy for practical computations, and all the terms of order higher than zero with respect to the deformational (bulk) degrees of freedom are dropped. So we obtain the equation for the angular momentum of a purely rigid body:

$(\dot{\nabla} \boldsymbol{\omega})=\int_{V^{\prime}} \mathbb{O} \mathbf{X} \times \mathbf{F}_{e x t} d v+\int_{\partial V^{\prime}} \mathbb{O} \mathbf{X} \times \mathbf{R} d s$

This drastic simplification is justified by the fact of small perturbation allowed for the solid. Numerical tests will show satisfactory results for this simplified equation within our framework of assemblies of very stiff objects. For interested readers, the equation with one order higher, i.e. linearized, is derived in Appendix A.

We can now write dynamics formulation for the deforming motion. Recall that consideration of dynamics in the local frame, as opposed to the global one, introduces fictitious forces (Feynman et al., 2013). Denote by $\tilde{\omega}=\mathbb{O}^{T} \omega, \widetilde{\dot{\omega}}=\mathbb{O}^{T} \dot{\omega}$ the standard geometric vector transformations from the global frame to the local one, and $\mathbb{T}$ denotes the Cauchy stress tensor in the rigid frame of reference. Then the momentum equation in the local frame is

$\rho \dot{\mathbf{v}}^{\prime}=\nabla \cdot \mathbb{T}+\mathbb{O}^{T} \mathbf{F}_{\text {ext }}+\mathbf{F}_{t r}+\mathbf{F}_{\text {rot }}$,

where

$\mathbf{F}_{t r}=-\mathbb{O}^{T} \rho \dot{\mathbf{v}}_{C}$

$\mathbf{F}_{\text {rot }}=-\tilde{\boldsymbol{\omega}} \times\left(\tilde{\boldsymbol{\omega}} \times \rho \mathbf{X}^{\prime}\right)-\widetilde{\dot{\omega}} \times \rho \mathbf{X}^{\prime}-2 \tilde{\boldsymbol{\omega}} \times \rho \mathbf{v}^{\prime}$

and the divergence operator $\nabla \cdot$ is taken with respect to the actual configuration $\mathbf{x}^{\prime}$. The term $\mathbf{F}_{t r}$ is a translational (rectilinear) inertia force and distributed homogeneously over the whole solid, acting as an additional gravity pointing in the direction opposite to the solid's rectilinear acceleration. In computations, this term is better written in terms of external forces and contact due to (11). The term $\mathbf{F}_{\text {rot }}$ consists of rotational fictitious forces, namely, the centrifugal, Euler and Coriolis forces.

Note that the Equations (11) and (12) require only the time discretization, while Equation (14) is the one requiring both temporal and FEM discretization. The latter one provides the most computational load. In this paper, elasticity law $\mathbb{T}=\mathbb{C}: \mathbb{E}$ with linear strain tensor $\mathbb{E}$ is imposed on the stress tensor since the deformations are assumed to be of small magnitude in the local frame. The nonlinear with respect to displacement and velocity terms are neglected. This lets us use time-independent volume $V$ and its surface $\partial V$ instead of $V^{\prime}$ and $\partial V^{\prime}$, as well as ignoring the difference between $\rho$ and $\rho_{R}$ in various integrations performed in the Finite Element discretization. We should obtain the standard formulation of the elastic problem with constant in time stiffness matrix.

As we have finished with the formalism, it is now possible to construct a consistent numerical method for solving (10) using the FFR method.

\section{The numerical method}

Since a body is provided in its rigid (undeformed) state at the initial instant of time, it is straightforward to construct unique operators $\mathbb{L}$ and $\mathbb{G}$ for it, so this allows to find $\mathbf{v}_{R}=\mathbb{G} \mathbb{v}$ and, consequently, $\mathbf{v}_{D}$. From it, we easily obtain $\boldsymbol{v}^{\prime}$ using (9). The timestep is denoted $h$ and is assumed constant in this paper. The value of a discrete function $f$ at a node $i$, corresponding to $t_{i}=t_{0}+h i$, is denoted $f_{i}$.

\subsection{Single solid}

Traditionally, in non-smooth contact dynamics (NSCD) all the contact interactions are represented as those occurring through a finite set of points rather than continuum surfaces, as the last case lacks consistent general framework due to its difficulty. The nonsmooth contact framework relies on contact laws that are written for each point individually, together organizing a contact network with a global contact law. A commonly used one and employed in this paper is the law connecting the reaction $\mathbf{R}$ at the current contact point with the relative velocity $\mathbf{U}$ of the interacting solids at that point, for example, the Signorini non-penetration condition and the Coulomb friction law with a friction coefficient $\mu$, respectively below:

$\left\{R_{N}=\operatorname{proj}_{\mathbb{R}_{+}}\left(R_{N}-c_{N} g\right)\right.$

$\left\{\mathbf{R}_{T}=\operatorname{proj}_{D\left(\mu R_{N}\right)}\left(\mathbf{R}_{T}-c_{T} \mathbf{U}_{T}\right)\right.$.

Here subscripts $N$ and $T$ mean normal and tangential components respectively, $g \geq 0$ is the gap function, i.e. the distance between the bodies examined for contact, $c$ means a positive conditioning coefficient, and $D(r)$ means a two-dimensional disk of radius $r$. Note that $\mathbf{R}_{T}$ is parallel to $U_{T}$ and is pointing in the opposite direction. Symbol $\operatorname{proj}_{A}$ denotes projection onto convex set $A \in \mathbb{R}^{k}$ containing zero, for some $k$. This means the following: $x \in A$ implies $\operatorname{proj}_{A} x=x$ and $x \notin A$ implies $\operatorname{proj}_{A} x=c x$ where $c$ is the largest such number that $c x \in A$. For more on this, as well as a more used Signorini velocity condition, see (Moreau, 1988; Moreau 2004; Jean, 1999; Vola et al., 1998).

For practical purposes, at least for one body in contact, a contact point that this structure is having at a certain instant of time is assumed to be precisely a node of the mesh arising from its space discretization, such as the Finite Element discretization. Then for the second body, since the actual contact region in general is not localized close enough to either of the FEM nodes on its surface, the position of its contact point is found from the orthogonal projection of the point of the first body to the surface of the second.

Let the body have a set $\{\alpha\}$ of contact points on its surface over the time interval $\left[t_{i}, t_{i+1}\right]$. There is an orientation at the contact point $\alpha$ given via a matrix $\mathbb{C}_{\alpha}$ that is constructed from its basis vector being orthogonal to the surface of the body at the contact point. So we assume by default that at least one of the bodies has a smooth (in geometrical sense) surface at the contact point during the 
contact in order for $\mathbb{C}_{\alpha}$ to be defined correctly. The reaction at the point $\alpha$ expressed in $\mathbb{C}_{\alpha}$ is denoted $\tilde{\mathbf{R}}_{\alpha}$. So in the global frame $\mathbf{R}_{\alpha}=\mathbb{C}_{\alpha} \tilde{\mathbf{R}}_{\alpha}$. Note that in general $\mathbb{C}_{\alpha} \neq \mathbb{O}$. There is also a strong assumption that the contact geometry does not change during the time interval $\left[t_{i}, t_{i+1}\right]$, which is equivalent to the fact that $\mathbb{C}_{\alpha}$ remains constant over the interval and the point $\alpha$ does not move in the local frame. Nevertheless, to achieve as much generality as possible, we assume that $\mathbb{O}$ may be changing over that same interval $\left[t_{i}, t_{i+1}\right]$. This may happen, for example, when a body turns while touching a static surface with its corner.

We shall consecutively discretize (11), (13) and (14). The scheme used for the temporal discretization is a Newmark symmetric one (also called trapezoidal), known for being a second-order accurate (for smooth regimes), unconditionally stable implicit scheme, (Hughes, 1987; Ascher and Petzold, 1998). For (11), obtain

$\mathbf{v}_{C, i+1}=\mathbf{v}_{C, i}+\frac{h}{2 m} \int_{V}\left(\mathbf{F}_{e x t, i}+\mathbf{F}_{e x t, i+1}\right) d v+\frac{h}{m} \sum_{\alpha} \mathbb{C}_{\alpha} \tilde{\mathbf{R}}_{\alpha}$.

For the rotational motion (13), obtain

$$
\begin{aligned}
\mathbb{J}_{i+1} \omega_{i+1}= & \mathbb{J}_{i} \omega_{i}+\frac{h}{2} \int_{V} \mathbb{O}_{i} \mathbf{X} \times \mathbf{F}_{\text {ext, } i} d v+\frac{h}{2} \int_{V} \mathbb{O}_{i+1} \mathbf{X} \times \mathbf{F}_{\text {ext }, i+1} d v \\
+ & h \mathbb{O}_{i+1} \sum_{\alpha} \mathbf{X}(\alpha) \times \mathbb{O}_{i+1}^{T} \mathbb{C}_{\alpha} \tilde{\mathbf{R}}_{\alpha} .
\end{aligned}
$$

Write the time-stepping equation for the deforming motion (14) as

$$
\begin{aligned}
\rho \mathbf{v}_{i+1}^{\prime}= & \rho \mathbf{v}_{i}^{\prime}+\frac{h}{2}\left(\nabla \cdot \mathbb{T}_{i}+\nabla \cdot \mathbb{T}_{i+1}\right)+\frac{h}{2}\left(\mathbb{O}_{i}^{T} \mathbf{F}_{\text {ext }, i}+\mathbb{O}_{i+1}^{T} \mathbf{F}_{\text {ext }, i+1}\right) \\
& -\frac{h}{2} \frac{\rho}{m} \int_{V}\left(\mathbb{O}_{i}^{T} \mathbf{F}_{\text {ext }, i}+\mathbb{O}_{i+1}^{T} \mathbf{F}_{\text {ext }, i+1}\right) d v-h \frac{\rho}{m} \mathbb{O}_{i+1}^{T} \\
& \times \sum_{\alpha} \mathbb{C}_{\alpha} \tilde{\mathbf{R}}_{\alpha}+\frac{h}{2}\left(\mathbf{F}_{\text {rot }, i}+\mathbf{F}_{\text {rot }, i+1}\right) .
\end{aligned}
$$

The two terms with the integration and the summation are the inertia force $\mathbf{F}_{t r}$ due to translation, expressed through the external and contact forces respectively. From (15)-(17), we see that the only way the elastic degrees of freedom influence the degrees of freedom of the rigid part is through contact forces. If no contact interactions occur, the rigid part becomes fully decoupled from the local elasto-dynamics and obeys the momentum conservation due to external forces only. This will also be confirmed by the numerical experiments in Section 4.

An interesting observation about a typical structure we consider is that a low magnitude of its perturbations makes it possible to neglect the contribution from the local displacement $\mathbf{d}^{\prime}$ and its velocity $\mathbf{v}$ in all three terms inside $\mathbf{F}_{\text {rot }}$, leaving only the contribution coming from the rigid configuration $\mathbf{X}$. This in particular completely eliminates the Coriolis term and leaves

$$
\begin{aligned}
\mathbf{F}_{r o t}= & -2 \tilde{\boldsymbol{\omega}} \times \rho \mathbf{v}^{\prime}-\tilde{\boldsymbol{\omega}} \times\left(\tilde{\boldsymbol{\omega}} \times \rho\left(\mathbf{X}+\mathbf{d}^{\prime}\right)\right)-\tilde{\boldsymbol{\omega}} \times \rho\left(\mathbf{X}+\mathbf{d}^{\prime}\right) \approx-\tilde{\boldsymbol{\omega}} \\
& \times(\tilde{\boldsymbol{\omega}} \times \rho \mathbf{X})-\tilde{\boldsymbol{\omega}} \times \rho \mathbf{X} .
\end{aligned}
$$

This is a very crucial advantage of the FFR method for very stiff objects, since FEM does not only create a constant in time stiffness matrix in this case, but also a constant mass matrix, so the inversion of a generalized matrix (see below) is simplified significantly. This makes it different from the applications of flexible multi-body dynamics, where mass matrices end up being coordinate- dependent, (Shabana et al., 2007). We emphasize that this simplification is due to conditions $\left|\mathbf{d}^{\prime}\right| \ll 1$ and $\left|\mathbf{v}^{\prime}\right| \ll 1$ and otherwise could lead to inaccurate prediction. Note that for the centrifugal force its trapezoidal discretization is obviously taken as the average between two endpoints of the interval $\left[t_{i}, t_{i+1}\right]$ as opposed to the Euler force. The latter is approximated via the mid-point rule due to impossibility to approximate the angular acceleration at the endpoints:

$$
-h \widetilde{\dot{\omega}} \times \rho \mathbf{X} \approx-\rho\left(\mathbb{O}_{i+1}^{T} \omega_{i+1}-\mathbb{O}_{i}^{T} \omega_{i}\right) \times \mathbf{X} .
$$

Let $\left\{\mathbf{u}_{j}\right\}$ with $j=1, \ldots, n$ denote the set of the basis nodal functions for the whatever Finite Element discretization we are using inside the local frame $C X Y Z$, and the local displacement $\mathbf{d}^{\prime}$ and velocity $\mathbf{v}$ are approximated as $\sum \psi_{j} \mathbf{u}_{j}$ and $\sum \lambda_{j} \mathbf{u}_{j}$ respectively, with $n$ unknowns $\psi_{j}$ and $\lambda_{j}=\dot{\psi}_{j}$. Sं o (17) bears its FEM analogue with a mass matrix $\mathbb{M}$ and a stiffness matrix $\mathbb{K}$ in a form

$$
\begin{aligned}
\left(\mathbb{M}+\frac{h^{2}}{4} \mathbb{K}\right) \lambda_{i+1}= & \left(\mathbb{M}-\frac{h^{2}}{4} \mathbb{K}\right) \lambda_{i}-h \mathbb{\psi _ { i }}+\frac{h}{2}\left(\left(\mathbf{F}_{e}\right)_{i}+\left(\mathbf{F}_{e}\right)_{i+1}\right) \\
& +h \mathbf{A}+\mathbb{A}\left(\mathbb{O}_{i}^{T} \omega_{i}-\mathbb{O}_{i+1}^{T} \omega_{i+1}\right)+h \\
& \times \sum_{\alpha} \overline{\mathbb{H}}_{\alpha}^{T} \mathbb{O}_{i+1}^{T} \mathbb{C}_{\alpha} \tilde{\mathbf{R}}_{\alpha} .
\end{aligned}
$$

Here

$$
\begin{aligned}
\mathbb{A} & =\int_{V} \rho_{R}\left(\mathbf{X} \times \mathbf{u}_{1}, \quad \mathbf{X} \times \mathbf{u}_{2}, \quad \cdots, \quad \mathbf{X} \times \mathbf{u}_{n}\right)^{T} d v, \\
\mathbf{A} & =\int_{V} \rho_{R}\left(\mathbf{u}_{1} \otimes \mathbf{X}: \mathbb{S}+s \mathbf{X}^{T} \mathbf{u}_{1}, \mathbf{u}_{2} \otimes \mathbf{X}: \mathbb{S}+s \mathbf{X}^{T} \mathbf{u}_{2}, \cdots, \mathbf{u}_{n} \otimes \mathbf{X}\right. \\
& \left.: \mathbb{S}+s \mathbf{X}^{T} \mathbf{u}_{n}\right)^{T} d v, \\
\mathbb{S} & =-\frac{1}{2}\left(\mathbb{O}_{i}^{T} \omega_{i} \otimes \omega_{i} \mathbb{O}_{i}+\mathbb{O}_{i+1}^{T} \omega_{i+1} \otimes \omega_{i+1} \mathbb{O}_{i+1}\right), \\
s & =\frac{1}{2}\left(\left|\omega_{i}\right|^{2}+\left|\omega_{i+1}\right|^{2}\right) .
\end{aligned}
$$

The term $\mathbf{F}_{e}$ accounts both for the point-wise external force and its contribution inside $\mathbf{F}_{t r}$. Its $j$-th component is

$\mathbf{F}_{e, j}=\mathbb{O}^{T}:\left(\int_{V} \mathbf{u}_{j} \otimes \mathbf{F}_{e x t} d v-\frac{1}{m} \int_{V} \rho_{R} \mathbf{u}_{j} d v \otimes \int_{V} \mathbf{F}_{e x t} d v\right)$.

$\mathbf{F}_{e}=0$ for homogeneous in space external force, such as, for example, gravity. Similar to the external forces, the point-wise contact force and its contribution to the translational inertia term $\mathbf{F}_{t r}$ are organized together, via the matrix $\overline{\mathbb{H}}_{\alpha}$ given as

$$
\begin{aligned}
\overline{\mathbb{H}}_{\alpha}= & \mathbf{u}_{1}(\alpha)-\frac{1}{m} \int_{V} \rho_{R} \mathbf{u}_{1} d v, \quad \mathbf{u}_{2}(\alpha)-\frac{1}{m} \int_{V} \rho_{R} \mathbf{u}_{2} d v, \cdots, \quad \mathbf{u}_{n}(\alpha) \\
& \left.-\frac{1}{m} \int_{V} \rho_{R} \mathbf{u}_{n} d v\right) .
\end{aligned}
$$

The non-smooth contact dynamics framework connects the local degrees of freedom, for which the dynamics equations are 
formulated, with the actual ones, used for the formulation of the physical interaction laws between the solids. For the dynamics equations, two generalized mass matrices, explicit and implicit, uniting both the rigid and the deforming parts are introduced:

$\mathbb{A}_{ \pm}=\left(\begin{array}{ccc}m \rrbracket_{3 \times 3} & 0 & 0 \\ 0 & \rrbracket_{i+\frac{1}{2} \pm \frac{1}{2}} & 0 \\ 0 & 0 & \mathbb{M} \pm \frac{h^{2}}{4} \mathbb{K}\end{array}\right)$.

In order to further integrate the presented FFR method into the NSCD framework, the evaluation of the relative velocity $\mathbf{U}_{\alpha}$ in the contact frame $\mathbb{C}_{\alpha}$ must be presented through the local variables $\mathbf{v}_{C}$, $\omega, \lambda$. This evaluation is given by (8):

$\mathbb{C}_{\alpha} \mathbf{U}_{\alpha}=\mathbf{v}_{C}+\boldsymbol{\omega} \times \mathbb{O} \mathbf{X}(\alpha)+\mathbb{O} \mathbf{v}^{\prime}(\alpha)+\boldsymbol{\omega} \times \mathbb{O} \mathbf{d}^{\prime}(\alpha)$.

The last term is nonlinear with respect to the local variables, since the local displacement $\mathbf{d}_{i+1}^{\prime}$ is implicitly predicted through $\lambda_{i+1}$. In this article, it is dropped due to the large stiffness assumption $\mathbf{v}_{D} \approx \mathbb{O} \mathbf{v}^{\prime}$. The numerical tests with neglected nonlinear term show physically consistent results for moderate spins, as they will be compared with the method of a large transformation. So we shall obtain the extended to the rigid part matrices $\mathbb{H}_{\alpha \pm}$ so that the explicit and the implicit relative velocities are

$\left(\mathbf{U}_{\alpha}\right)_{i+\frac{1}{2} \pm \frac{1}{2}}=\mathbb{H}_{\alpha \pm}\left(\begin{array}{c}\mathbf{v}_{C} \\ \boldsymbol{\lambda}\end{array}\right)_{i+\frac{1}{2} \pm \frac{1}{2}}$

with a hybrid term

$\left.\mathbb{H}_{\alpha \pm}=\mathbb{C}_{\alpha}^{T} \mathbb{O}_{i+\frac{1}{2} \pm \frac{1}{2}} \quad \mathbb{O}_{i+\frac{1}{2} \pm \frac{1}{2}}^{T}, \quad-\mathbb{X}(\alpha) \mathbb{O}_{i+\frac{1}{2} \pm \frac{1}{2}}^{T}, \quad \overline{\mathbb{H}}_{\alpha}\right)$.

Here $\mathbb{X}$ represents a skew-symmetric matrix made of the vector $\mathbf{X}$

Remark 3. Rigorously speaking, the term $\overline{\mathbb{H}}_{\alpha}$ inside $\mathbb{H}_{\alpha \pm}$ defined by (20) should not contain the inertia part (the integral terms in (20)) which is only used for correct distribution of the translational inertia force, arising from the reaction, over the whole FEM variables. In (21), this part produces $\int_{V} \rho_{R} \sum_{j} \lambda_{j} \mathbf{u}_{j} d v$ which is a FEM approximation of $\int_{V} \rho_{R} \mathbf{v}^{\prime} d v$, but since $\mathbf{v}_{D}$ is treated as $\mathbb{O} \mathbf{v}^{\prime}$, according to Section 2.1, this term may be seen as zero due to $\llbracket \mathbf{v}_{D}=0$.

So the motion of a single body is formalized by a nonlinear system of equations

$\mathbb{A}_{+}\left(\begin{array}{c}\mathbf{v}_{C} \\ \omega \\ \lambda\end{array}\right)_{i+1}=\mathbb{A}_{-}\left(\begin{array}{c}\mathbf{v}_{C} \\ \omega \\ \lambda\end{array}\right)_{i}+h \mathbf{F}_{g}+h \sum_{\alpha} \mathbb{H}_{\alpha+}^{T} \tilde{\mathbf{R}}_{\alpha}$

with vector $\mathbf{F}_{g}$ containing the remaining non-contact terms of (15), (16) and (19) respectively. For the position variables, we shall have trapezoidal approximations in a form

$\mathbf{x}_{C, i+1}=\mathbf{x}_{C, i}+\frac{h}{2}\left(\mathbf{v}_{C, i}+\mathbf{v}_{C, i+1}\right)$

$\psi_{i+1}=\psi_{i}+\frac{h}{2}\left(\lambda_{i}+\lambda_{i+1}\right)$

Let $\mathbb{Q}$ denote a skew-symmetric matrix of the angular velocity:
$\mathbb{Q}=\left(\begin{array}{ccc}0 & -\omega_{z} & \omega_{y} \\ \omega_{z} & 0 & -\omega_{y} \\ -\omega_{y} & \omega_{x} & 0\end{array}\right)$

From the discretization of $\dot{\mathbb{O}}=\mathbb{Q} \mathbb{O}$, (Cardona and Geradin, 2001), we get

$\mathbb{O}_{i+1}=\left(\llbracket-\frac{h}{2} \mathbb{Q}_{i+1}\right)^{-1}\left(\llbracket+\frac{h}{2} \mathbb{Q}_{i}\right) \mathbb{O}_{i}$.

\subsection{Multi-body system with contact}

If all $\tilde{\mathbf{R}}_{\alpha}$ in (23) were known, this system of $6+n$ equations for each time step could be solved with some iterative solver for nonlinear systems. The information about $\tilde{\mathbf{R}}_{\alpha}$ can only be obtained via coupling with interaction laws, arising in non-smooth multibody dynamics. Consider a system of $N$ bodies, and for each body the Equation (23) is used. Let us introduce $0.5 \leqslant \theta \leqslant 1$. Uniting dynamics equations together with the connection between the generalized velocities and the actual relative velocities at contacts, we obtain a global system for the multi-body dynamics

$\left(\begin{array}{cc}\mathbb{A}_{+} & -h \mathbb{H}_{+}^{T} \\ \theta \mathbb{H}_{+} & 0\end{array}\right) \cdot\left(\begin{array}{c}\mathbf{V}_{i+1} \\ \mathbf{R}\end{array}\right)=\left(\begin{array}{l}\mathbb{A}_{-} \\ -(1-\theta) \mathbb{H}_{-}\end{array}\right) \cdot \mathbf{V}_{i}+\left(\begin{array}{l}h \mathbf{F}_{g} \\ \mathbf{U}_{i+\theta}\end{array}\right)$,

$\operatorname{Law}\left(\mathbf{U}_{i+\theta}, \mathbf{R}\right)=0$

to solve over $\left[t_{i}, t_{i+1}\right]$, with $\mathbb{A}_{ \pm}$and $\mathbf{F}_{g}$ now meaning their global analogues, $\mathbf{V}$ and $\mathbf{R}$ contain all the degrees of freedom for all bodies (generalized velocity vector) and all the relative reactions respectively. The relative velocity $\mathbf{U}_{i+\theta}$ and $\mathbf{R}$ are conntected via some interaction law (26). Note that if the Signorini non-penetration condition is used in (26), the parameter $\theta$ serves as a measure of restitution during shocks, ranging from $\theta=0.5$ (full restitution) to 1 (sticking).

Global matrices $\mathbb{H}_{ \pm}$are comprised of $\mathbb{H}_{\alpha \pm}$ in a following way. In the beginning of each time step, the whole multi-body system is scanned for potential contact interactions. The network of contact points is only initialized for those surface FEM nodes of each solid that pass criteria of close enough proximity to another solid in the system, for the sake of reducing the size of matrices $\mathbb{H}_{+}$. These contact points shall be called active. Matrix $\mathbb{H}_{+}$is made of special block rows. The entire contact network is naturally divided into local interactions for each pair of the solids, and if a local interaction is taking place for some fixed pair of bodies $l$ and $m$, this corresponds to a certain and only one block row of matrix $\mathbb{H}_{+}$. Each block row of such type consists of zeros and only two matrices of type $\mathbb{H}_{\alpha+}$, placed at block columns $l$ and $m$. This follows from the fact that the relative velocity for two interacting solids at their common contact point $\alpha$ is

$\left(\mathbf{U}_{\alpha}\right)_{i+1}=\mathbb{H}_{\alpha+}^{l}\left(\begin{array}{c}\mathbf{v}_{C} \\ \omega \\ \lambda\end{array}\right)_{i+1}^{l}-\mathbb{H}_{\alpha+}^{m}\left(\begin{array}{c}\mathbf{v}_{C} \\ \omega \\ \lambda\end{array}\right)_{i+1}^{m}$

or with opposite sign, depending on which body is considered 'master' or 'slave' with respect to another. Note that $\mathbb{H}_{\alpha+}^{l}$ and $\mathbb{H}_{\alpha+}^{m}$ may have a different number of columns, which depends on the number of the Finite Element degrees of freedom each of the bodies is using, but they necessarily have the same number of rows, which 
is equal to 3. $\mathbb{H}_{-}$is simply an explicit modification of $\mathbb{H}_{+}$corresponding to the orientation matrices $\mathbb{O}_{i}$ of each body in the network.

The Schur complement system, (Koziara and Bicanic, 2008), in this case is formed as

$\left\{\begin{array}{l}\mathbf{U}_{i+\theta}=\mathbb{W}\left(\mathbf{V}_{i+1}\right) \mathbf{R}+\mathbf{U}_{\text {free }}\left(\mathbf{V}_{i+1}\right), \\ \mathbf{V}_{i+1}=\mathbb{B}_{1}\left(\mathbf{V}_{i+1}\right) \mathbf{R}+\mathbf{B}_{2}\left(\mathbf{V}_{i+1}\right)\end{array}\right.$

where

$$
\begin{aligned}
& \mathbb{B}_{1}=h \mathbb{A}_{+}^{-1} \mathbb{H}_{+}^{T}, \\
& \mathbf{B}_{2}=\mathbb{A}_{+}^{-1} \mathbb{A}_{-} \mathbf{V}_{i}+h \mathbb{A}_{+}^{-1} \mathbf{F}_{g}, \\
& \mathbb{W}=\theta \mathbb{H}_{+} \mathbb{B}_{1}, \\
& \mathbf{U}_{\text {free }}=\theta \mathbb{H}_{+} \mathbf{B}_{2}+(1-\theta) \mathbb{H}_{-} \mathbf{V}_{i} .
\end{aligned}
$$

The dependence of $\mathbb{W}, \mathbf{U}_{\text {free }}$ on $\mathbf{V}_{i+1}$ implies that the contact solver for 27(a) must be used simultaneously along with the nonlinear dynamics solver in 27 (b), such as, for example, a fixedpoint method. There exists a variety of contact solvers, of which the most known are the nonlinear Gauss-Seidel method (NGLS) and the Newton's method, (Koziara and Bicanic, 2008). The latter is usually faster, but is not robust and may not converge for highly dynamic applications. We shall use NGLS in the numerical section due to its robustness, proven convergence results, (Jourdan et al., 1998). A brief sketch of the algorithm used in the numerical section is the following.

Concerning implementation, note the algorithmic block called 'Rotator'. It updates all the variables for both iterative solvers due to rotations only. Global mass matrices $\mathbb{A}_{ \pm}$are time-dependent only due to presence of the inertia tensors $\sqrt{ }$. The inversion of $\mathbb{A}_{+}$and the following multiplication $\mathbb{A}_{+}^{-1} \mathbb{A}_{-}$for each step are computationally cheap due to the block-diagonal profile of these matrices. Indeed,

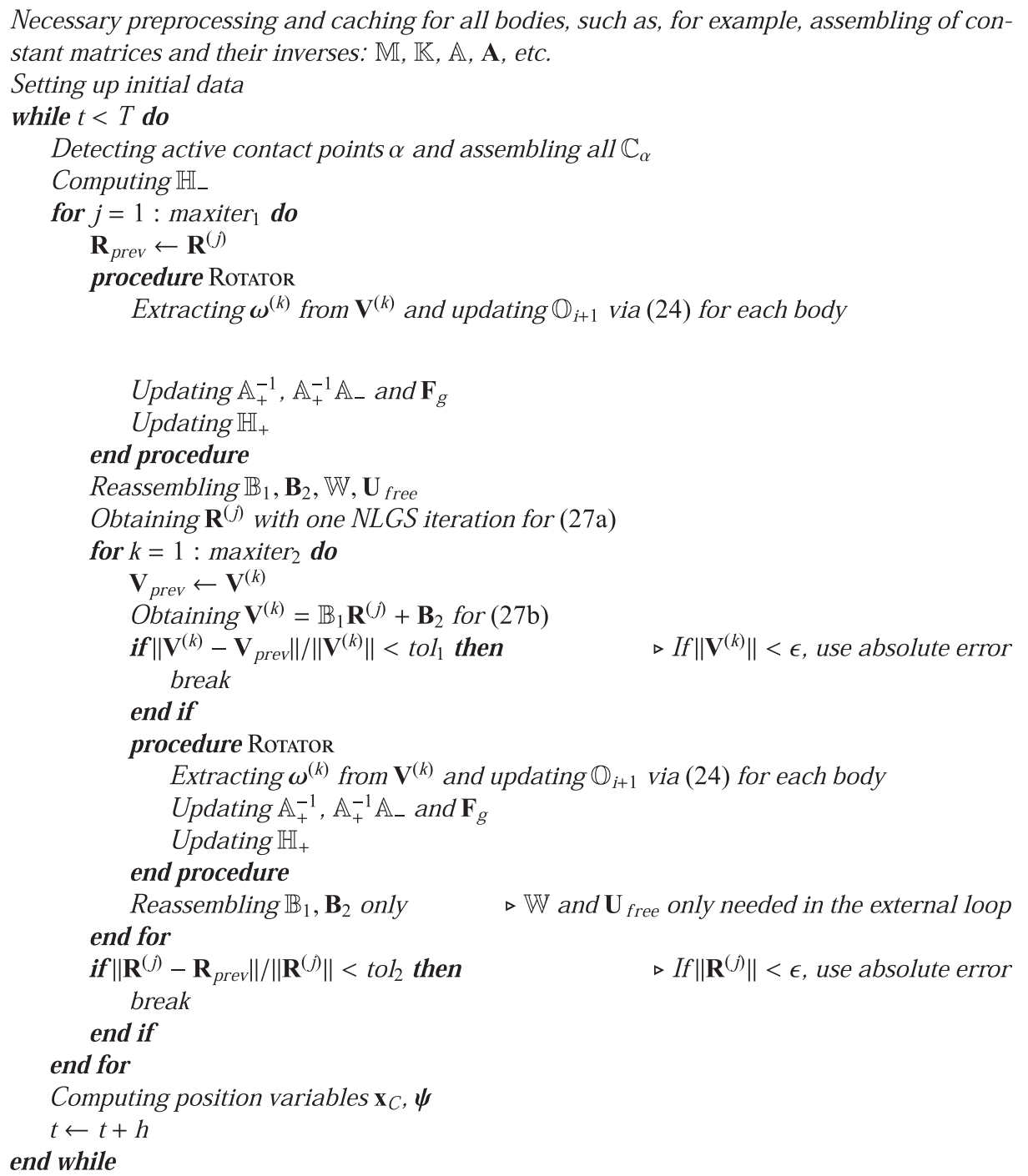


for an arbitrary body its inertia tensor satisfies $\rrbracket=\mathbb{O}_{D} \mathbb{O}^{T}$, where $\rrbracket_{D}$ is a diagonal form of the inertia tensor with respect to the floating rigid frame, so it is constant and so is its inverse. Thus for a single solid

$$
\mathbb{J}_{i+1}^{-1}=\mathbb{O}_{i+1} \rrbracket_{D}^{-1} \mathbb{O}_{i+1}^{T}
$$

and

$$
\mathbb{J}_{i+1}^{-1} \rrbracket_{i}=\mathbb{O}_{i+1} \rrbracket_{D}^{-1} \mathbb{O}_{i+1}^{T} \mathbb{O}_{i} \rrbracket_{D} \mathbb{O}_{i}^{T} .
$$

\section{Numerical experiments}

To evaluate the method of a floating frame of reference, numerical tests were conducted for two cases. The first one represents a single object subjected to finite rigid motion with no contact interactions. In the second, we perform simulations for three objects undergoing contact. The simulations were carried out with fixed time step $\Delta t=10^{-4}$ in all of the methods and scenarios. For implementation, we utilized Armadillo $-\mathrm{C}++$ linear algebra library.

\subsection{No contact}

A rectangular cuboid of dimensions $1 \times 1 \times 2$ is made of stone of uniform density $\rho=2500 \frac{\mathrm{kg}}{\mathrm{m}^{3}}$ and stiffness parameters $E=35 \mathrm{GPa}$ and $\nu=0.2$, where $E$ and $v$ denote Young's modulus and Poisson's ratio respectively, Fig. 3. Initially, the faces of the cuboid are parallel to either planes $O x y, O x z, O y z$, with $z$-axis being parallel to the longest edge of the cuboid, and its center of mass is placed at the origin. The cuboid is uniformly discretized with a trilinear hexahedral element, (Hughes, 1987), as $4 \times 4 \times 8$, giving rise to 225 nodes.

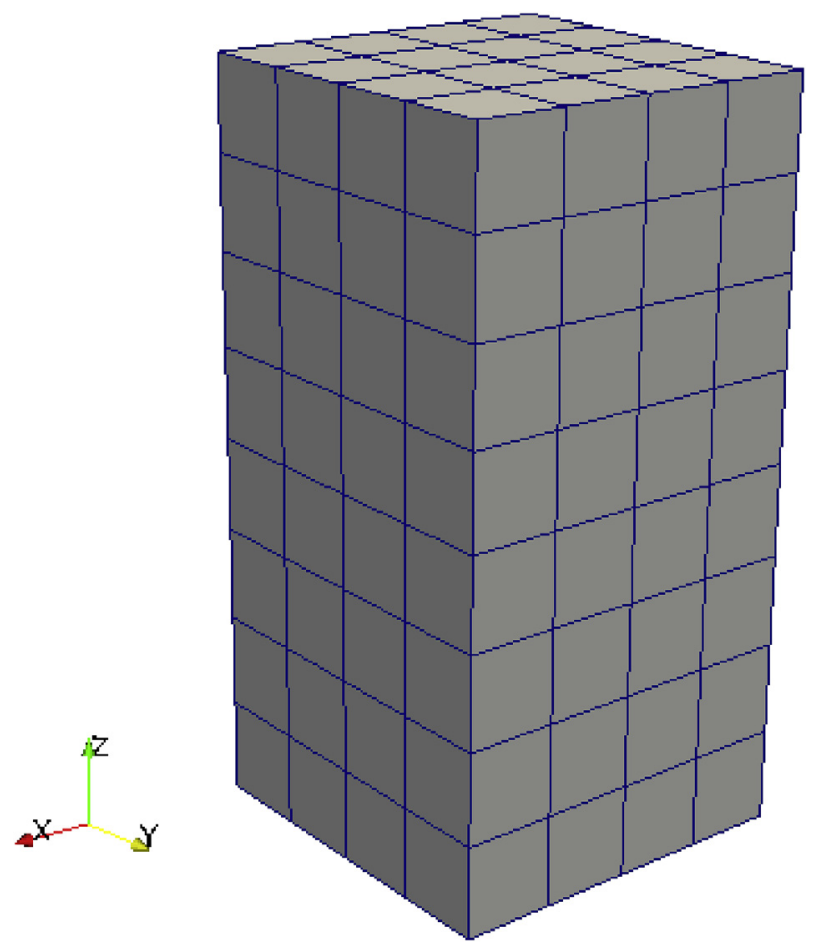

Fig. 3. Solid for simulation.
Here, our main goal is to compare the accuracy and performance of the FFR method with existing methods of small deformation and a large transformation, when used to simulate the dynamics of the cuboid subjected to initial velocity as of a rigid body. No external forces or contact interactions assumed. The initial conditions were given as

$\mathbf{v}_{C, 0}=\left(\begin{array}{l}1 \\ 0 \\ 0\end{array}\right), \omega_{0}=\left(\begin{array}{c}0 \\ \omega_{0} \\ 0\end{array}\right)$

where we try separately $\omega_{0}=1$ and $\omega_{0}=4 \pi$. The cuboid will move in the direction of $x$-axis, while rotating around its center line parallel to the $y$-axis. It should make two full turns within $1 \mathrm{~s}$ in the second case.

The total number of the degrees of freedom is equal to $6+225 \times 3=681$, of which 6 correspond to the velocity of the center of mass and the angular velocity. For the large transformation method, i.e. Total Lagrangian method, we use nonlinear weak formulation with test function $\xi$ in the way shown:

$\int_{V} \rho \frac{\partial \mathbf{v}}{\partial t} \xi+\int_{V} \mathbb{E}(\lambda \operatorname{tr}(\mathbb{E}) \llbracket+2 \mu \mathbb{E}): \nabla \xi=0$,

with transformation gradient $\mathbb{F}=\frac{\partial \mathbf{X}}{\partial \mathbf{X}}$, Green-Lagrangian strain tensor $\mathbb{E}=\frac{1}{2}\left(\mathbb{F}^{T} \mathbb{E}-\mathbb{q}\right)$, and velocity field $\mathbf{v}(\mathbf{X}, t)$ being given in the global frame. Standard Finite Element discretization in space and Newmark discretization in time of this weak form bear nonlinear algebraic system at every time step with $225 \times 3=675$ degrees of freedom:

$$
\begin{aligned}
\left(\mathbb{M}+\frac{h^{2}}{4} \mathbb{K}\right) \lambda_{i+1}= & \left(\mathbb{M}-\frac{h^{2}}{4} \mathbb{K}\right) \lambda_{i}-h \mathbb{K} \psi_{i}-\frac{h}{2} \\
& \times\left(\mathbf{n}\left(\lambda_{i}\right)+\mathbf{n}\left(\psi_{i}+\frac{h}{2}\left(\lambda_{i}+\lambda_{i+1}\right)\right)\right),
\end{aligned}
$$

with $\psi$ and $\lambda$ now meaning the absolute nodal displacement and velocity respectively. The solution of this system is incorporated in the form of the internal loop in Algorithm 1, either as a fixed-point method or Newton method. Finally, for the small deformation method, we only remove nonlinear terms $\mathbf{n}$ from (28), leaving the rest the same, and apply the fixed-point solver providing the solution at the first iteration. For the stopping criteria in the both nonlinear solvers and the contact solver in Algorithm 1 we used tolerance of $10^{-7}$.

We monitor the $x$-displacement $d_{x}$ of the point whose initial coordinates are $(0,0,1)^{T}$, performing $10^{4}$ time steps until $T=1$. If the body was treated as purely rigid, then the displacement would satisfy $d_{x}(t)=t+\sin \left(\omega_{0} t\right)$. This trajectory is used as a reference for all the methods, due to large enough a stiffness of the solid. The graphs are shown on Fig. 4. Both the FFR method and the large transformation method with Newton's nonlinear solver show excellent match with the trajectory from the purely rigid case. The small deformation method fails to represent rotation due to inability to track geometric nonlinearity, and provides linear in time displacement. The large transformation method with fixedpoint nonlinear solver manages to recover accurate trajectory only within a limited number of time steps. The number of iterations per time step tends to grow and eventually reaches the maximum allowed number of 500. These time instances are indicated with vertical lines in both figures.

We remark that we also experimented with neglect of the rotational fictitious forces in FFR model, i.e. the term $\mathbf{F}_{\text {rot }}$ in (14). The displacement of the rigid frame was calculated the same as 

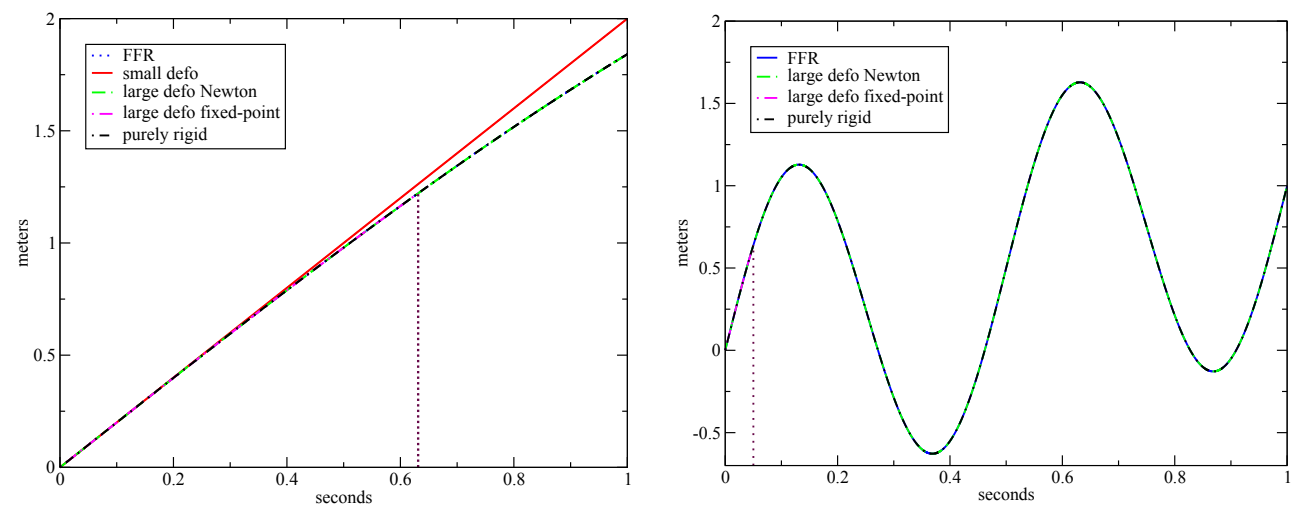

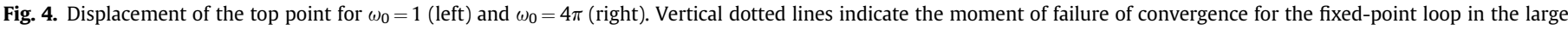
transformation method.

previously up to machine precision, and all the local oscillations were reduced to zero $\left(\lambda_{i}=\psi_{i}=0\right.$ in (19)). This agrees with the fact that due to the absence of contact interactions, the dynamics of the rigid part decouples from the FEM degrees of freedom in (23). This is a consequence from the small deformability assumption that we employed when deriving (13). On the other hand, if additionally $\mathbf{F}_{\text {ext }}=0$, then $\mathbf{F}_{\text {rot }}$ is the only term containing infromation about the rigid motion that influences the dynamics in the local frame in this case.

Finally, we address performance of the FFR method compared to other formulations by presenting the total runtime for each of the succeeded methods in Fig. 5. The first four measurements correspond to $\omega_{0}=1$ and the other four to $\omega_{0}=4 \pi$.

Both the FFR and the Total Lagrangian methods required 2 iterations of the internal loop per time step, but the computational disadvantage of the Total Lagrangian model is apparent. It is justified by the necessity to assemble jacobian matrix and solve a linear system at every iteration of the Newton's method, which sacrifices performance. Contrary to the large transformation method, the floating frame method works more efficiently since the stiffness matrix is evaluated once in the local frame before the start of the time evolution, and the only nonlinearity left in the scheme is due to rotations, which appears to be easily handled by a cheap fixed-

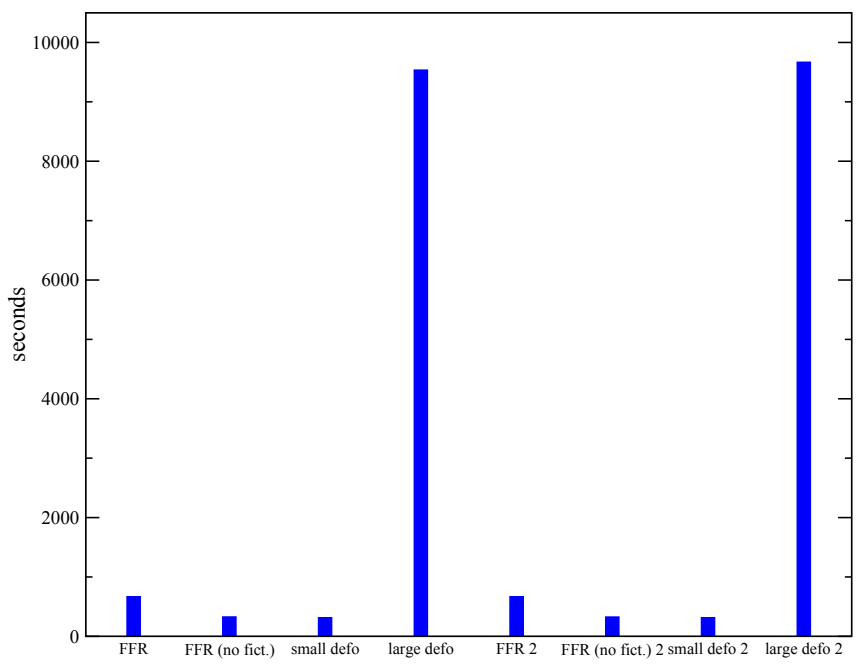

Fig. 5. Runtime in seconds: number ' 2 ' denotes the simulation corresponding to $\omega_{0}=4 \pi$. Both 'large defo' cases refer to the Total Lagrangian approach with the Newton method as a nonlinear sover point solver. The FFR method and the small deformation method showed to have comparable computational expenses, with only marginally better performance for the latter. The FFR formulation with neglected fictitious forces required only 1 fixed-point iteration, while the original FFR method required 2, which explains why it took less elapsed computational time for the first one to complete.

\subsection{With contact}

Three cuboids of dimensions $1 \times 1 \times 2$ and $1 \times 0.8 \times 2$ for the upper one are placed on an absolutely rigid surface that can move along the $x$-axis which is initially orthogonal to the largest vertical surface of the upper block. The initial positions of the center of mass are $(0,2 / 3,1)^{T},(0,-2 / 3,1)^{T}$ and $(0,0,3)^{T}$. Refer to Fig. 6. Previous material parameters are used for the three cuboids, and they are uniformly discretized in a way analogous to that in the previous computational study. There are 70 contact points in total for the

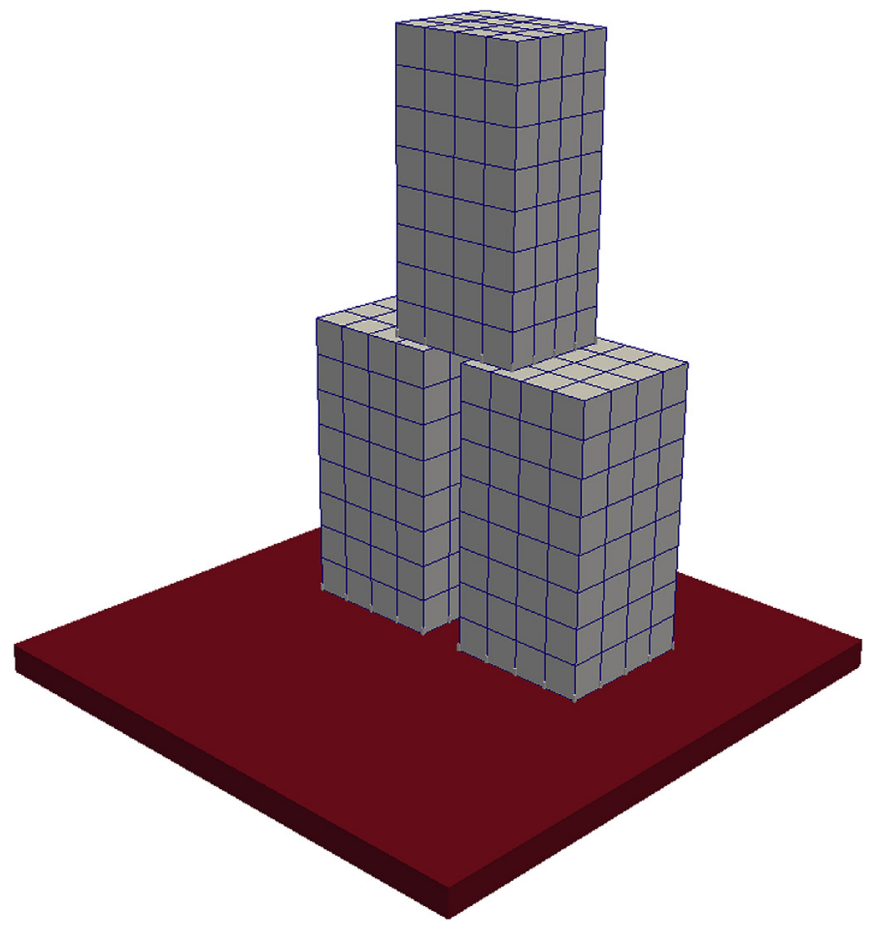

Fig. 6. Solids for simulation. 
simulation, 10 between each of the lower solids and the upper one, and 25 between each of the lower solids and the rigid surface. The Signorini velocity condition (Moreau, 1988) and the Coulomb friction laws are used at contacts, $\theta=1$. The friction coefficients $\mu=0.5$ at the rigid surface and $\mu=0.3$ between the upper solid and the lower ones. The simulation was carried out until $T=1.5$, while a horizontal earthquake was imposed for the rigid surface in the direction of $x$-axis via a velocity law

$$
v= \begin{cases}0 & \text { if } t \leqslant 0.1 \\ 0.9 \sin \left(\frac{5}{3} \pi(t-0.1)\right) & \text { if } t>0.1\end{cases}
$$

Damping was added to each of the solids in form of a matrix $\mathbb{D}=10^{-4} \mathbb{K}$.

We again compare the presented method of a floating frame of reference with the methods of small deformation and a large transformation employing the Newton's nonlinear solver in the internal loop. The nonlinear Gauss-Seidel contact solver was used in all three. The tolerance of $10^{-5}$ was imposed for the relative error in the reaction $\mathbf{R}$ in the external loop and in the generalized velocity $\mathbf{V}_{i+1}$ in the internal loop of Algorithm 1. For all three simulations, the convergence was normally being reached after 3 iterations in the internal loop and after at most 16 iterations in the NLGS in the external loop.

The time-dependent graph of the average of the displacement over the upper cuboid's top surface obtained from all the three methods is shown on Fig. 7. The lowest curve corresponds to the displacement of the rigid surface. The displacement of the top surface of the upper cuboid lags behind because the solid inclines in the opposite direction due to the large inertia force.

It is clearly seen the FFR method again provides a much better accuracy compared to the small deformation approach which does not work reliably for finite rotations. The relative error in the displacement between the curves obtained via the large transformation method and the FFR method is $2.1 \%$, compared to $37.6 \%$ coming from the small deformation method. The computational efficiency of the FFR method was again similar to that of the small deformation method and significantly stood out compared to the large transformation approach.

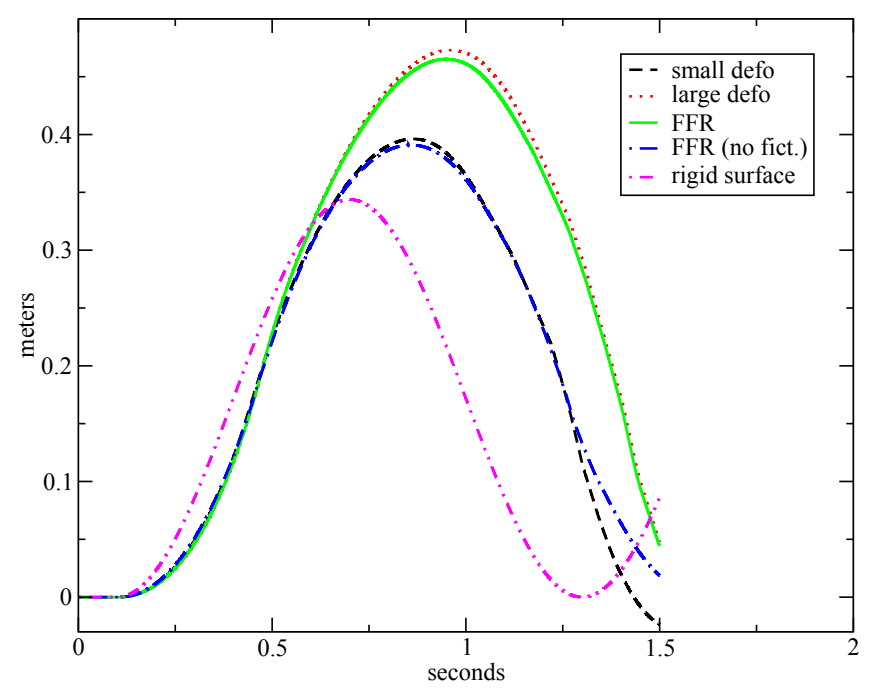

Fig. 7. Mean displacement of the top surface of the upper block in the direction of the earthquake.
The FFR method was also tested with the term $\mathbf{F}_{\text {rot }}$ set to 0. To reach the mentioned tolerance of $10^{-5}$, only 2 iterations in the fixed-point loop and no more than 11 iterations in the NLGS were required. It is clearly seen that the neglect of that term even for stiff solids distorts retults visibly if contact interactions are present. For better accuracy it must remain at place. In Fig. 7, the solution curve in this case initially resembles that produced by the small deformation method.

\section{Conclusion}

In the preceding, a numerical method based on the method of a floating frame of reference to solve dynamics of a system assuming deformability of low magnitude and non-smooth contact interactions was formulated. The main goal of the method is to conduct separation between the rigid and the deforming motions at each step in a way that minimizes the coupling between the reference (rigid) motion and the bulk behavior of the system with respect to this frame of reference. In our results, we found the following.

- For a deformable solid under rotation, there is always a geometrically nonlinear deformation with respect to the reference frame that we introduced. However, it is negligible if large stiffness is assumed. For this reason, constant in time stiffness matrix was proposed.

- The minimal coupling was achieved thanks to the zeroth order (with respect to the FEM degrees of freedom) approximation in the angular momentum equation and neglect of the FEM degrees of freedom in the fictitious forces in the reference frame. The latter granted a constant mass matrix.

- The neglect of the geometrically nonlinear dynamics in the reference frame also allowed for linear connection between the generalized velocity and the relative velocity at contact points, Equation (21).

- The method provides better experimental results for stiff systems undergoing small deformations than that provided by the method involving absolute nodal coordinate fomulation with constant stiffness matrix and, compared to the Total Lagrangian approach (no linearization used), works faster since the linear stiffness model holds in the rigid frame. For this FFR model, the rigid (reference) dynamics fully decouples from the bulk (local) dynamics in case of free motion, i.e. the absence of contact forces.

The next step in the research would be an introduction of some model reduction technique for the deforming part of the motion residing in the rigid frame only, in order to diminish the size of problem (25) and provide even better speed-up with insignificant sacrifice of accuracy, depending on use cases. An example of such work is given in (Lozovskiy, 2014).

\section{Acknowledgements}

1. The authors want to thank V. Acary and M. Jean for the fruitful discussions, as well as S. Pagano and L. Daridon for sharing their thoughts and advice. This work has been supported by French National Research Agency (ANR) through COSINUS program (project SALADYN n ${ }^{\circ}$ ANR-08-COSI-014).

\section{Appendix A}

Let $D$ denote a determinant (jacobian) of the transformation gradient matrix 
$D=\operatorname{det}\left[\frac{\partial \mathbf{x}^{\prime}}{\partial \mathbf{X}}\right]$

Then $\rho_{R}=\rho D$. Differentiate (6) in time. This produces two terms on the right-hand side (RHS), according to Leibniz's rule:

$(\rrbracket \boldsymbol{\omega})=\int_{V}\left((\boldsymbol{\omega} \times \mathbb{O} \mathbf{X}) \times \rho_{R} \mathbf{v}\right) d v+\int_{V} \mathbb{O} \mathbf{X} \times \rho_{R} \dot{\mathbf{v}} d v$.

The second term on the RHS may be written for the actual domain $V^{\prime}$ and

$$
\int_{V^{\prime}} \mathbb{O} \mathbf{X} \times \rho \dot{\mathbf{v}} d v^{\prime}=\int_{V} \mathbb{O} \mathbf{X} \times \mathbf{F}_{e x t} D d v+\mathbb{O} \int_{V^{\prime}} \mathbf{X} \times \nabla \cdot \mathbb{T} d v^{\prime} .
$$

Consider

$$
\int_{V^{\prime}} \mathbf{X} \times \nabla \cdot \pi d v^{\prime}=-\sum_{j=1}^{3} \int_{V^{\prime}} \frac{\partial \mathbf{X}}{\partial x_{j}^{\prime}} \times \pi \mathbf{e}_{j} d v^{\prime}+\int_{\partial V^{\prime}} \mathbf{X} \times \pi \mathbf{n} d s
$$

with $\mathbf{e}_{j}$ meaning unit $j$-axis vector and $\mathbf{n}$ the outward unit normal to the surface of $\partial V^{\prime}$. Linearization provides

$$
-\sum_{j=1}^{3} \int_{V^{\prime}} \frac{\partial \mathbf{X}}{\partial x_{j}^{\prime}} \times \pi \mathbf{e}_{j} d v^{\prime}=0
$$

and so

$$
\text { (1) } \int_{V^{\prime}} \mathbf{X} \times \nabla \cdot \llbracket d v^{\prime}=\int_{\partial V^{\prime}} \mathbb{O} \mathbf{X} \times \mathbf{R} d s \text {. }
$$

It also can be shown directly that, up to the linear order of the bulk degrees of freedom, $D=1+\nabla_{R} \cdot \mathbf{d}^{\prime}$. So we obtain

$$
(\rrbracket \boldsymbol{\omega})=\int_{V}\left(1+\nabla_{R} \cdot \mathbf{d}^{\prime}\right) \mathbb{O} \mathbf{X} \times \mathbf{F}_{e x t} d v+\int_{\partial V^{\prime}} \mathbb{O} \mathbf{X} \times \mathbf{R} d s+\rrbracket^{\prime} \boldsymbol{\omega}
$$

with tensor

$$
\rrbracket^{\prime}=\int_{V} \mathbb{O} \mathbf{X} \otimes \rho_{R} \mathbf{v}_{D} d v-\int_{V}(\mathbb{O} \mathbf{X})^{T} \rho_{R} \mathbf{v}_{D} d v \rrbracket_{3 \times 3} .
$$

The first term on the RHS of (A.29) is equal to $\rrbracket^{\prime} \omega$.

\section{Remark 4. Define}

$$
\mathbb{G}^{T}=\left(\begin{array}{c}
\int_{V}(\cdot) d v \\
\int_{V} \mathbf{r} \times(\cdot) d v
\end{array}\right) .
$$

Note in a case of a finite discrete mechanical system, $\mathbb{G}^{T}$ is a usual transposition of matrix $\mathbb{G}$. The left-hand side term of $(A .2)$ is a continuous analogue of the rotational part of quantity $\mathbb{G}^{T} \mathbf{F}_{\text {int }}$ which can be written explicitly for discrete systems. This quantity is precisely zero for purely rigid bodies, due to symmetry of the stress tensor (in the absence of force couples), and may serve as a measure of deformability of a solid.

\section{References}

Acary, V., Brogliato, B., 2008. Numerical Methods for Nonsmooth Dynamical Systems; Applications in Mechanics and Electronics. Lect Notes in Appl and Comput Mech 35. Springer, Germany.

Agrawal, O., 1984. Application of Deformable Body Mean Axis to Flexible Mechanical Systems. Ph. D. Thesis. Department of Mechanical Engineering, University of Illinois, Chicago, IL.

Alart, P., 2014. How to overcome indetermination and interpenetration in granular systems via nonsmooth contact dynamics. An exploratory investigation. Comput. Methods Appl. Mech. Eng. 270, 37-56.

Alsafadie, R., Hjiaj, M., Battini, J.-M., 2010. Corotational mixed finite element formulation for thin-walled beams with generic cross-section. Comput. Methods Appl. Mech. Eng. 199 (49-52), 3197-3212.

Areias, P., Garção, J., Pires, E.B., Barbosa, J.I., 2011. Exact corotational shell for finite strains and fracture. Comput. Mech. 48 (4), 385-406.

Ascher, U.M., Petzold, L.R., 1998. Computer Methods for Ordinary Differential Equations and Differential-algebraic Equations. Society for Industrial and Applied Mathematics, USA.

Banton, J., Villard, P., Jongmans, D., Scavia, C., 2009. Two-dimensional discrete element models of debris avalanches: parametrisation and the reproducibility of experimental results. J. Geophys. Res. Solid Earth 114, 15.

Belytschko, T., Hsieh, B.J., 1973. Nonlinear transient finite element analysis with convected coordinates. Int. J. Numer. Methods Eng. 7, 255-271.

Belytschko, T., Liu, W.K., Moran, B., 2000. Nonlinear Finite Elements for Continua and Structures. Wiley, England.

Bergan, P., Horrigmoe, G., 1976. Incremental variational principles and finite element models for nonlinear problems. Comp. Methods Appl. Mech. Eng. 7, 201-217.

Cardona, A., Geradin, M., 2001. Flexible Multibody Dynamics: a Finite Element Approach. Wiley, England.

Crisfield, M., 1990. A consistent corotational formulation for nonlinear threedimensional beam elements. Comput. Methods Appl. Mech. Eng. 81, $131-150$.

Cundall, P., Hart, R., 1992. Numerical modelling of discontinua. Eng. Comput. 9 (2), $101-113$.

Debnath, L., Mikusinski, P., 2005. Introduction to Hilbert Spaces with Applications Academic press.

Devloo, P., Geradin, M., Fleury, R., 2000. A corotational formulation for the simulation of flexible mechanisms. Multibody Syst. Dyn. 4 (2-3), 267-295. http:// dx.doi.org/10.1023/A:1009884131140.

Escalona, J., Valverde, J., Mayo, J., Domnguez, J., 2003. Reference motion in deformable bodies under rigid body motion and vibration. Parts I \& II. J. Sound Vib. 264 (5), 1045-1072.

Felippa, C.A., Haugen, B., 2005. A unified formulation of small-strain corotational finite Elements: I. Theory. Comput. Methods Appl. Mech. Eng. 194, 2285-2335.

Feynman, R., Leighton, R., Sands, M., 2013. The Feynman Lectures on Physics Desktop Edition. Basic Books. Volume I.

Ghaboussi, J., Barbosa, R., 1990. Threedimensional discrete element method for granular materials. Int. J. Numer. Anal. Meth. Geomech. 14 (7), 451-472.

Goldstein, H., Poole, C., Safko, J., 2014. Classical Mechanics, Pearson New International Edition. Pearson Higher Ed.

Hughes, T.J.R., 1987. The Finite Element Method: Linear Static and Dynamic Finite Element Analysis. Englewood Cliffs, USA.

Jean, M., 1999. The non-smooth contact dynamics method. Comput. Methods Appl. Mech. Eng. 177, 235-257.

Jourdan, F., Alart, P., Jean, M., 1998. A Gauss-seidel like algorithm to solve frictional contact problems. Comput. Methods Appl. Mech. Eng. 155, 31-47.

Koziara, T., Bicanic, N., 2008. Semismooth Newton method for frictional contact between pseudo-rigid bodies. Comput. Methods Appl. Mech. Eng. 197 (33-40), 2763-2777.

Landau, L., Lifshitz, E., 1976. Mechanics, third ed. Pergamon Press.

Lozovskiy, A., 2014. The modal reduction method for multibody dynamics with nonsmooth contact. Int. J. Numer. Methods Eng. 98 (13), 937-959.

Manzella, I., Labiouse, V., 2009. Flow experiments with gravels and blocks at small scale to investigate parameters and mechanisms involved in rock avalanches. Eng. Geol. 109, 146-158.

Mishra, B., Rajamani, R., 1992. The discrete element method for the simulation of ball mills. Appl. Math. Model. 16 (11), 598-604.

Moreau, J.J., 1988. Unilateral Contact and Dry Friction in Finite Freedom Dynamics, Non-smooth Mech and Appl 302. CISM Courses and Lectures, pp. 1-82.

Moreau, J.J., 2004. An Introduction to Unilateral Dynamics, Novel Approaches in Civil Engineering. Lect Notes in Appl and Comput Mech 14. Springer, Berlin, pp. $1-46$.

Rankin, C., Brognan, F., 1986. An element-independent corotational procedure for the treatment of large rotations. ASME J. Press. Vessel Technol. 108, 165-174.

Rankin, C., Nour-Omid, B., 1988. The use of projectors to improve finite element performance. Comput. Struct. 30, 257-267.

Schwertassek, R., Wallrapp, O., Shabana, A., 1999. Flexible multibody simulation and choice of shape functions. Nonlinear Dyn. 20 (4), 361-380.

Shabana, A., Schwertassek, R., 1998. Equivalence of the floating frame of reference approach and finite element formulations. Int. J. Nonlinear Mech. 33 (3), $417-432$ 
Shabana, A., Bauchau, O., Hulbert, G., 2007. Integration of large deformation finite element and multibody system algorithms. J. Comput. Nonlinear Dyn. 2. 351-359.

Simo, J.C., 1985. A finite strain beam formulation. Part I: the three-dimensional dynamic problem. Comp. Methods Appl. Mech. Eng. 49, 55-70.
Veubeke, B.F., 1976. The dynamics of flexible bodies. Int. J. Eng. Sci. 14, 895-913. Vola, D., Pratt, E., Jean, M., Raous, M., 1998. Consistent time discretization for a dynamical frictional contact problem and complementarity techniques. Rev. Eur. Des. Elem. Finis. 7, 149-162. 